\title{
Drug Targets and Biomarker Identification from Computational Study of Human Notch Signaling Pathway
}

\author{
Saikat Chowdhury ${ }^{1,2}$ and Ram Rup Sarkar ${ }^{1,2 *}$
}

${ }^{1}$ Chemical Engineering and Process Development Division, CSIR-National Chemical Laboratory, Pune, Maharashtra, 411008, India

${ }^{2}$ Academy of Scientific \& Innovative Research (AcSIR), New Delhi, 110 001, India

\begin{abstract}
Notch signaling pathway is widely implicated in controlling various cellular functions, cell fate determination, and stem cell renewal in human but aberrant activity in cancer stem cells may cause different types of cancers. Understanding the complexity of this pathway to identify important targets for cancer therapy and to suppress the pathway activity without affecting the normal functions is of utmost importance to clinical and experimental pharmacologists. For developing therapeutic strategy, non availability of detailed molecular interactions, complex regulations and cross talks with other pathways pose a serious challenge to get a coherent understanding of this pathway. This motivated us to reconstruct the largest human cell specific Notch pathway with more number of molecules and interactions available from literatures and databases. To identify probable drug targets and biomarkers for cancer prognosis, we also performed computational study of the pathway using structural and logical analysis and identified important hub proteins, cross talks and feedback mechanisms. The model simulation is validated using reported mRNA expression profile in Glioblastoma cell line and the predictions not only show significant accuracy but also able to identify the undetermined expressions. From our simulation, to identify novel combinations of drug targetable proteins and better substitute for GAMMA SECRETASE inhibition, we proposed two alternative scenarios: partial suppression of Notch target proteins by NICD1 \& HIF1A; and complete suppression by NICD1 \& MAML, in Glioblastoma cell line. This reconstructed Notch signaling pathway and the computational analysis for identifying new biomarkers and combinatory drug targets will be useful for future in-vitro and in-vivo analysis to control different cancers.
\end{abstract}

Keywords: Notch signaling pathway; Reconstruction; Structural and logical analysis; Glioblastoma; Drug targets and biomarkers identification

\section{Introduction}

Notch signaling pathway is a conserved developmental pathway which is involved in cell fate determination at the stage of embryonic development and also plays important roles in tissue and organ development, hematopoiesis, vascular growth etc., [1-3]. Mostly activated by the membrane associated ligands of juxtaposed cells, this pathway then triggers most of the cellular functions including transcription of various genes, cell cycle progression, anti-apoptosis and simultaneously regulates other signaling pathways through the cross talks $[4,5]$. Activation of this pathway requires the involvement of two nearly adjacent cells, one of which acts as a transducer of the signal and the other receives and processes that signal [6] along with several intermediate reactions and further production of Notch intracellular domain (NICD) [6-9]. Translocation of NICD into the nucleus induces various cellular functions including cell division, cell cycle progressions, cell growth etc., through transcription of various Notch target genes and activation of other signaling and metabolic pathways in cell [10-24]. Hence, activation of this pathway is utmost important in stem cell development and cellular growth, and should be highly tuned with the activity of other pathways [25].

Sometimes this concerted process is found to work in a wrong direction [25-27], and failures in its normal functional activities can cause development of various types of cancers, such as, glioblastoma, breast cancer, osteosarcoma, prostate cancer and melanoma [28,29]. Most of the cancer cell lines have shown significant level of upregulation of its activator proteins (Onco-proteins) and down regulations of its tumor suppressor proteins [30]. The "gain or loss" of functions of these Notch pathway associated proteins have clearly proved its correlation with cancer development, and hence can be used as a biomarker for cancer diagnosis. Various molecular biology experiments have also shown that inhibition of the activators of this pathway can drastically reduce the cancer progression in different stages [31], hence identification of drug targetable proteins and their small molecule inhibitors in the pathway to reduce various types of cancer development has always been an important field of research to the pharmacists and clinical biologists [31]. Recent identification of GAMMA SECRETASE as a probable drug target in the pathway is found to reduce the Notch pathway activity by not allowing it to cleave the Notch receptor in the membrane [32]. But, unfortunately the compound Semagacestat (LY450139), which inhibits the GAMMA SECRETASE and was in the Phase III trial of Alzheimer's disease, failed to meet the desired goal as it was compromising with several risk factors including Skin cancer [33]. Also, the drugs developed against other target molecules, such as NOTCH1, NOTCH4, DLL4, NRARP etc., have not shown desirable result due to their toxicity and side effects [31]. Hence, inhibition of Notch pathway is now under the inspection of several cancer pathologists and the merits of targeting the Notch pathway have raised numerous questions as certain imbalance of this pathway can impose long term side effects such as, gastrointestinal toxicity and diarrhea [34].

On the other hand, identification of suitable and alternative drug targets for inhibition of this pathway in various cancers including Glioblasotma is undoubtedly useful and effective for cancer therapy

*Corresponding author: Ram Rup Sarkar, Senior Scientist, Chemical Engineering and Process Development, CSIR-National Chemical Laboratory, Pune 411008, Maharashtra, India, Tel: +91-2590-3040; Fax: +91-20-2590 2621; E-mail rr.sarkar@ncl.res.in

Received September 29, 2013; Accepted October 22, 2013; Published October 29, 2013

Citation: Chowdhury S, Sarkar RR (2013) Drug Targets and Biomarker Identification from Computational Study of Human Notch Signaling Pathway. Clin Exp Pharmacol 3: 137. doi:10.4172/2161-1459.1000137

Copyright: (c) 2013 Chowdhury S, et al. This is an open-access article distributed under the terms of the Creative Commons Attribution License, which permits unrestricted use, distribution, and reproduction in any medium, provided the original author and source are credited. 
[35], but requires the understanding of the exact mechanisms that are governing the normal functions of Notch signaling pathway in functional cells. Numerous experiments on different regulations, feedback loops, and cross talks of this pathway have been reported time to time by several research groups $[16,21,22,25]$. But unfortunately, the integrations of these experimental findings have not been performed properly and none of the signaling pathway database provides this extensive and up to date information and hence it has become impossible to predict the consequence of the inhibition of this pathway in a diseased situation. Moreover, study of the effects of several drug targets from a population of large number of proteins is also difficult through in-vitro and in-vivo analysis. Recent advancements in computational approaches, bioinformatics tools, and mathematical methods have contributed immensely in the understanding and analysis of large signaling pathways [36-38] and were useful to answer several biological questions in signaling systems including identification of important molecules/ proteins as well as alternative combinatorial drug targets for Glioma, Colon and Pancreatic cancer [39]. Among several computational tools, structural analysis using graph theoretical methods [40] and logical analysis using Boolean formalisms $[39,41,42]$ have shown promising results for visual and/ or topological interpretation of a very large complex network and identification of important target proteins, particularly when the kinetic parameters for pathway reactions are not available. Unfortunately, very few computational studies have been performed on Notch signaling pathway to study the dynamic of the network, but none of the studies addressed this kind of problems [43-45].

This article is specifically addressed to study the complexity of the human cell specific Notch signaling pathway and to identify aa well as propose alternative solutions to control cancer situation. For this, we have curated a most up-to-date and comprehensive human cell specific Notch signaling pathway interactions data by assembling the experimental results found from numerous literatures and signaling pathway databases. Using this collated information, we have reconstructed a new Notch signaling pathway map with 115 molecules and 231 interactions or reactions, the largest number of molecular entities and their corresponding interactions to the best of our knowledge. Further, we have performed computational study using graph theoretical and logical analysis to model the reconstructed pathway and identify "Hub" proteins for alternative drug targets in place of GAMMA SECRETASE complex. Using the master logical model and varying the logical states of the input molecules of the pathway, we simulated different scenarios such as Normal Notch, Glioblastoma, GAMMA SECRETASE inhibition, and two proposed insilico combinatorial drug treated scenarios. From these two analyses we have been able to identify some important proteins, which have high centrality values and could be used as probable drug targets. In order to validate our in-silico logical model, the expression results obtained from the logical analysis have been compared with the expressions profile of mRNA found in experimental study on human Glioblastoma cell line [30].

The proposed model is able to predict the expression of the proteins with significant accuracy and also been able to predict the expression of some other proteins whose expressions were not obtained successfully in the experimental study on Glioblastoma cell line. Also, we are able to match the expressions of some Notch pathway proteins for GAMMA SECRETASE treated Glioblastoma cell with our in-silico model. From our perturbation study in the proposed drug treated scenario we have predicted two minimal and novel combinations of proteins, which are useful to suppress the expressions of Notch target proteins partially or completely in Glioblastoma cell model and may be used as better therapeutic targets for cancer. Before performing further in-vitro and in-vivo experiments, this model and computational study of the Notch signaling pathway is not only helpful for identification of drug targets and biomarkers for cancer prognosis but also show new direction to the clinical and experimental pharmacologists in a faster and cost effective way to identify the probable, and safe drug targets in-silico.

\section{Materials and Methods}

\section{Reconstruction of Notch Pathway Map}

In order to reconstruct the human cell specific Notch signaling pathway, we searched around 28 available and popular databases on cell signaling, protein-protein interaction, cancer pathway, and microarray expressions (Table S1). Although the data available in these databases are mostly scattered and heterogeneously presented (Table S2), but this extensive and elaborative database searching gave us the basic information of the pathway, core proteins and the connections among its associated proteins/ molecules, which are involved in the Notch signal transduction network and also its functional cross talks with other cell signaling pathways. Unfortunately there was no database available which gives complete and most up to date Notch pathway information along with cross talk molecules of other pathways. Therefore, in order to reconstruct a master pathway model of Notch signaling network, we used the core structure of Notch pathway available from the databases and collated additional information from different literatures and experimental reports (Table S1) to build a comprehensive, up to date and the largest human cell specific Notch signaling pathway to the best of our knowledge.

The Notch pathway shown in Figure 1 is completely based on manual curation of data from various databases and literature resources. In order to incorporate a new molecule or interaction, we set few criteria e.g., the newly inserted molecules should have at least one direct or indirect connection or interaction with the core Notch pathway molecules, all the newly inserted interactions should have at least one experimental evidence in a peer reviewed journal and all the molecules should be placed in the pathway map according to the specified locations i.e., extra-cellular and membrane region, cytoplasmic, nucleus and output. Different color codes were used to distinguish the protein molecules and interactions according to their locations and type of reactions respectively. The pathway map was drawn in CellDesigner Ver. 4.2, an open source "Systems Biology Marked Up Language" (SBML) based pathway illustrator software [46,47].

\section{Pathway drawing and annotation}

We first annotated the molecules of the pathway according to their sub cellular locations in the cell. In case of Notch signaling pathway we had to consider three sub-cellular locations: Extracellular and Membrane, Cytoplasm and Nucleus of Notch signal "Receiver Cell". In order to reduce the complexity and create a simple pathway map, we considered only these three sub-cellular locations, but one can also consider other sub-cellular locations such as Golgi body, Mitochondria, Endoplasmic reticulum etc.,. Moreover, as notch pathway is mostly activated by the ligands expressed by the neighboring cell, therefore we had to consider another cell membrane of Notch signal "Transmitter cell" to allocate the ligands [6]. In between these two membrane regions we also annotated a place for extracellular region.

In the Extracellular and Membrane region, we annotated 27 molecules including 4 Notch receptors (NOTCH1/2/3/4), 9 ligands 


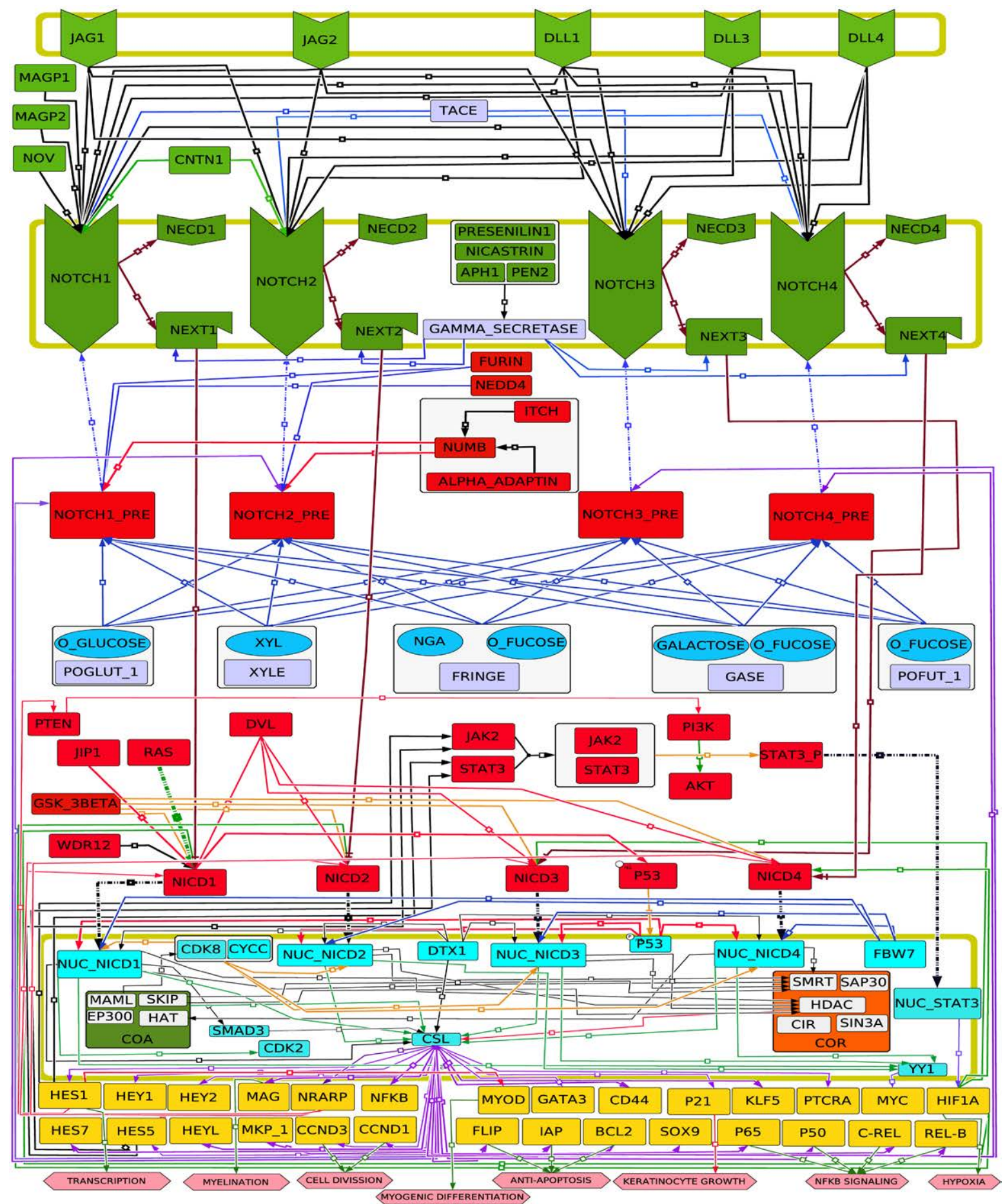

Figure 1: Reconstructed human cell specific Notch signaling pathway. See "Pathway Drawing and Annotation" part of the materials and method section for details of the color scheme used.

(JAG1/2, DLL1/3/4, MAGP1/2, NOV, CNTN1) molecules, 6 proteolytic enzyme complex including TACE, GAMMA SECRETASE complex etc., and the truncated portions of four Notch receptors (NEXT1/2/3/4 and NECD1/2/3/4) [48-61]. All the proteins (except metalloprotease enzymes TACE and GAMMA SECRETASE) of this location were colored as "Green" to distinguish these proteins from all other proteins in the network. In order to differentiate the proteins from all the membrane associated receptor proteins, the shape of the receptors proteins were chosen "Hexagonal" (a standard SBML CellDesigner notation) [46,47]. The metalloprotease enzymes of this location were colored "Light blue".

In the Cytoplasm region, we included total 35 molecules, out of which 5 molecules are metabolic compounds such as O-linked glucose, 
Xylose, O-linked Fucose, GALACTOSE, N-acetylglucosamine [6265]. The "Oval" shaped and deep blue colored entities were chosen to represent the metabolites in the pathway map. The color scheme of the enzyme molecules were kept same as in case of "Extracellular and Membrane" region. Besides these enzymes and metabolites, other cytoplasmic proteins (total 30) were colored as "Red" with rectangular shape.

The Nuclear region was annotated mostly with the transcription factors, co-activators and co-repressor molecules and complexes found at the time of data curation. There were 23 such proteins (NICD1/2/3/4, CSL, SMAD3 etc.,) and 2 transcription complexes (Co-activator and Co-repressor complex) considered [12-14]. The Co-activator complex (COA) and Co-repressor complex (COR) were colored as "Green" and "Orange", respectively. Other proteins were colored as "Dark Cyan". Also, in order to reduce the complexity we did not consider any gene or mRNA in this pathway map.

Moreover, except these three sub-cellular locations, we also annotated the Notch target proteins into a group called "Output". All the target proteins (total 28) in this group may belong to any sub-cellular locations depending on their functional activity. Few of them (e.g., HES and HEY proteins) are also the transcription factors for another genes [66]. All the proteins in this group are colored by "Yellow" color. We also linked these proteins with their phenotypic and functional activities (e.g., Transcription, Myelination, Cell Division, Anti-Apoptosis, Hypoxia etc.) [67,68]. Simultaneously, we also used specific color codes to distinguish the molecular reactions in the pathway on the basis of the type of the reactions. There were total 10 different types of reactions presented by colored arrows. Black: Physical interactions/ Complex Formation; Blue: Enzymatic reactions; Dotted Blue: Membrane Translocation; Brown: Proteolytic Cleavage; Red: Inhibition; Green: Activation; Dotted Green: Indirect Activation; Orange: Phosphorylation; Dotted Black: Nuclear Translocation; Violet: Protein Production, were considered at the time of drawing the pathway map.

\section{Structural analysis}

The structural and topological features of the reconstructed Notch pathway (Figure 1) were studied using 'Graph theory', which has shown promising results for visual and/ or topological interpretation of a very large complex network [39], despite its non-logical and static nature. The graph theoretical analysis was performed in open source software Gephi and igraph $[69,70]$. In order to identify the central nodes in the network, four types of centrality analysis were performed i.e., Degree centrality, Eigen vector Centrality, Closeness Centrality and Betweenness Centrality, and these were calculated using the inbuilt algorithms implemented in these software applications [69,70]. A detail description and definitions of the construction of Notch signaling network and the corresponding parameter values are given in the Supplementary file.

\section{Formation of logical model}

The logical relationships of the proteins were first prepared on the basis of biological and experimental knowledge about the pathway and its associated molecules. The entire logical model is available in the Supplementary file (Table S3). Standard logical operations AND, OR and NOT were used to form the logical equations and the procedure of formation of logical equations of the signaling pathway molecules was followed from previous study in this direction [39]. Total five different types of scenarios were created: Normal Notch Pathway
Scenario (NNS), Glioblastoma Scenario (GBS), Gamma Secreatase Inhibitor Scenario (GSI), and two proposed drug treated scenarios (TS1 and TS2). In NNS, we simulated the core Notch pathway scenario by considering the inputs of only the expression of core proteins of Notch pathway. On the other hand the Glioblastoma Scenario (GBS) was created by using the input of the expression values from mRNA expression data of Glioblastoma cell line [30]. The rest of the three scenarios were created by using the same logical states of the inputs of GBS with additional alterations/ perturbations of the logical states of the target proteins according to the need for the specific scenario and the respective simulated results of the output proteins were observed (Table S4 and Table S5). The entire simulation of logical model was performed in CellNetAnalyzer [42,71,72], a brief description of which is given in the Supplementary file.

\section{Results}

\section{Reconstructed Notch signaling network}

Pathway statistics: In this work, we have reconstructed a comprehensive, most up to date and largest human cell specific Notch signaling pathway to the best of our knowledge. The entire Notch pathway (Figure 1) was annotated and reconstructed manually by collating the data from various literatures, experimental findings and biological databases. Although the basic core pathway is same as the pathway map available in the existing signaling databases, but to the best of our knowledge the newly reconstructed pathway map consists most up-to-date information of interaction data which is not available in any freely available major academic databases. In this reconstructed pathway we included 115 molecules (96 core and 19 cross talking pathway molecules including proteins and organic compounds) and 231 molecular interactions. Different types of molecular reactions such as Physical interaction, Enzymatic reactions, Phosphorylation, Protein production, Activation, Inhibition, Nuclear translocation etc., were also considered to construct the pathway map. A comparison between this reconstructed Notch pathway data (i.e., molecules and interactions) with the pathway information from other major biochemical signaling databases (e.g., KEGG, Biocarta, Netpath etc.,) is presented in Table S2 of Supplementary file.

\section{Description of the reconstructed notch pathway}

Extracellular \& membrane reactions: Several experimental results have identified that all the four NOTCH receptors can express in the cell membrane of a Notch "Signal receiver" cell, whereas the membrane bound ligands JAG/DLL class proteins are expressed in the membrane of Notch signal "Transmitter cell" [6]. In the reconstructed pathway map (Figure 1), we have shown these proteins in the membrane regions of both cells. Moreover, besides these juxtracrine signaling property, Notch pathway can also be activated by the interactions between microfibrillar proteins MAGP1 and MAGP2, by CONTACTIN/ F3 (CNTN1) or by Nephroblastoma overexpressed (NOV) proteins [52-55] and were also shown separately in the extracellular regions in the pathway map. However, all these kind of ligand-receptors interactions are followed by the common protelytic cleavage of $\mathrm{NOTCH}$ receptors and subsequent formation of Notch Extracellular Domain (NECD) and Notch Extracellular Truncated Protein (NEXT) [6]. These proteolytic cleavage reactions were shown by brown arrows in the figure emanating from $\mathrm{NOTCH} 1 / 2 / 3 / 4$ receptor proteins. A metalloprotease enzyme TACE helps in this type of reactions to cleave the Notch receptors [7]. The blue arrows that are connecting TACE and the four NOTCH1/2/3/4 proteins; were used to show this enzymatic reaction in the pathway map (Figure 1 ). Subsequently, NEXT1/2/3/4 is 
again cleaved by another proteolytic enzyme GAMMA SECREATASE complex $[8,32]$, which was also included in the Figure 1.

Reactions in cytoplasm: Followed by the GAMMA SECRETASE mediated reactions, four NEXT proteins produce four homologues of Notch Intracellular Domains (NICD1/2/3/4) which then translocate into the cytoplasm and further moves towards the nucleus [6]. During this travel through cytoplasmic region, NICD encounters with various activator (RAS, GSK_3BETA, WDR12) and inhibitor (DVL, JIP1) proteins $[62,63,73,74]$. RAS pathway proteins activate NICD1 through some intermediate proteins which were not included in the pathway map for the sake of simplicity. This activation process was shown by dotted green arrow in Figure 1. Moreover the cytoplasmic region (or specifically the Golgi body) is also the place for post translation modification of Notch precursor proteins (NOTCH1_PRE, NOTCH2_ PRE, NOTCH3_PRE and NOTCH4_PRE) before they express in the cell membrane. In these reactions, notch precursors pass through several glycosylation or fucosylation reactions by Glucose, Galactose, Fucose and the enzymes POGLUT_1, FRINGE, GASE, POFUT_1etc.,. These post translational modifications of Notch precursors increase the specificity of ligand receptors interactions, so that it can easily recognize and interact with Notch ligands [75-78]. On the other hand Xylosylatin by Xylose with the help of the enzyme Xylosyltransferase (XYLE) is also observed in several cases which in turn reduce the specificity of notch ligand bindings [75]. All these enzyme-substrate reactions were included in the pathway map (Figure 1) and the connections were represented by blue colored arrows.

Reactions in nucleus: After encountering with several modifications and interactions by nuclear proteins, the activated NICD1/2/3/4 enters and starts the transcription process in the nucleus $[6,62,63,73,74]$. NICD initiates its transcription by binding with another transcription factor CSL, which in general forms a transcription repressor complex with another transcription Co-repressor complex (COR). It is a complex of SMRT, SAP30, HDAC, CIR, SIN3A proteins in the nucleus [11-14]. On the other hand, there is another protein complex which acts as a transcription co-activator of CSL to transcribe Notch target genes/ proteins such as HES1, HES5, HEY1, HEY2, HEYL, BCL2, P65, NOTCH1/2/3/4 etc., (colored as Yellow) [15-21]. Besides this CSL/ NICD/COR/COA mediated transcription, it is also reported that Notch pathway can also be activated through CONTACTIN/F3 (CNTN1) mediated interaction, which requires the involvements of DTX1as a transcription co-activator to produce the proteins MAG. This protein is involved in the oligodendrocyte maturation and myelination [54].

Cross talks with other pathways: Notch pathway has cross connections with different signaling pathways such as, JAK/STAT, PTEN/PI3K/AKT, RAS/MAPK, TGFB/SMAD3, CYCLIN/CDK, HYPOXIA/HIF1A, BCL2/IAP/ ANTI-APOPTOSIS, P65/P50/NFKB etc., which widen the scope of the study $[10,17,22,24,44,64,65,79-84]$. In our reconstructed Notch pathway (Figure 1), we tried to include more number of cross talks events with core proteins of Notch pathway. We included only those cross talk molecules of other pathways that had direct interaction/ influence on the core proteins of Notch pathway. For example, we added the cross talk of PTEN/ PI3K/AKT pathway in the pathway map. The output proteins HES1 or HES5 were found to inhibit directly the activity of PTEN protein which in normal situation inhibits the PI3K/AKT pathway activation. Therefore, inhibition of PTEN by Notch output proteins creates positive situation to activate the PI3K/AKT pathway in cell, which is also found to be associated with various cancer and tumorigenesis [84].

Feedback loops: In Notch pathway several feedback loops were identified that regulate and maintain its activity in various cellular situations and environmental stimuli. We found a cyclic feedback loop between an important protein of Hypoxia, HIF1A, to the Notch pathway proteins NICD, HES1, and HES5. From literature it is evident that HIF1A (a core protein of Hypoxia) can activate NICD1/2/3/4 which in turn helps to produce HES1/5 as well as the other Notch pathway target proteins [83]. The experimental findings also showed that these produced HES1/5 molecules can help to stabilize the JAK2/STAT3 complex formation and subsequent production of Phosphorylated STAT3 (STAT3_P) [84] and again helps to transcribe and activate HIF1A protein. Therefore, it can be easily conferred that the hypoxia situation (which is generally found in various cancer and tumor cell) further up-regulate the expression of various Notch target onco-genes/ proteins. A double negative feedback loop was also found in case of the cross talk with P53 pathway. It was found that the phosphorylated P53 inhibits NUC_NICD1/2/3/4 for its further transcription; on the other hand the phosphorylation of P53 is blocked by NICD1/2/3/4 in cytoplasm [63,65,80-82]. We assume that this double negative feedback loop helps to maintain the Notch pathway activation or inhibition under several pathological conditions and acts as a "Switch" for the production of Notch target proteins in cells. Besides, the precursor of NOTCH proteins are also the target output proteins of this pathway. Production of Notch molecules contributes a strong positive feedback effect in the entire network. We also found the presence of another strong negative feedback loop formed by NotchRegulated Ankyrin Repeat-containing Protein (NRARP), which was one of the Notch targeted output proteins. After production (by Notch regulated transcription factors such as NUC_NICD1/2/3/4, CSL, COA) it inhibits the NICD1/2/3/4 in the cytoplasm and reduces the active NICD into the nucleus so that further Notch regulated transcriptions can be stopped [85].

\section{Computational study of reconstructed Notch signaling pathway}

Network topology and structural analysis of Notch signaling pathway: The reconstruction of Notch signaling network inspired us to study its complex network structure and topology in the cell with the help of Graph theoretical analysis. In order to identify the important proteins/molecules that form "Hub" molecules in the network, we used the connectivity and centrality measurement parameters of the network such as Degree, Closeness, Betweenness, and Eigenvector centrality [39,86-88]. We calculated all these network parameter values for each protein of the network and extracted the significant proteins that had the parameter values higher than the corresponding average values. The values of network parameters are available in the "Supplementary" file. In Table 1, we have presented only the extracted and important proteins from the network analysis of Notch signaling pathway.

The extracted proteins enlisted in Table 1 helped us to identify the Hubs as well as the important centrally situated proteins from the network. In case of In-Degree, we identified almost all the four types of Notch receptors, Notch precursors and Notch Intracellular domains proteins were showing high In-Degree values compared to the other proteins in the network (more than the average value, 1.97). Again, out of those Notch receptor proteins, NOTCH1 had high values compared to the other homologues. Similarly, NOTCH1_PRECURSOR and NICD1 showed high values compared to their corresponding homologues present in the network. It signifies the importance of NOTCH1 compared to all other homologues as higher number of incoming connections or interactions are regulating this protein in the network (Figure S1 in Supplementary file, for In-Degree values). 


\begin{tabular}{|c|c|c|}
\hline $\begin{array}{l}\text { Network } \\
\text { parameters }\end{array}$ & $\begin{array}{l}\text { Average } \\
\text { value }\end{array}$ & Name of molecules \\
\hline In-degree & 1.97 & $\begin{array}{l}\text { NOTCH1, NOTCH2, NOTCH3, NOTCH4, GAMMA_SECRETASE, NICD1, NICD2, NICD3, NICD4, NOTCH1_PRE, NOTCH2_PRE, } \\
\text { NOTCH3_PRE, NOTCH4_PRE, NUC_NICD1, NUC_NICD2, NUC_NICD3, NUC_NICD4, CSL, COA, SMRT, COR, HDAC, YY1, } \\
\text { STAT3 }\end{array}$ \\
\hline Out-degree & 1.97 & $\begin{array}{l}\text { JAG1, JAG2, DLL1, DLL4, DLL3, TACE, GAMMA_SECRETASE, DVL, POGLUT1, O_GLUCOSE, POFUT_1, O_FUCOSE, XYLE, } \\
\text { XYL, GASE, GALACTOSE, FRINGE, NGA, GSK_3BETA, P53_P, NUC_NICD1, NUC_NICD2, NUC_NICD3, NUC_NICD4, CSL, } \\
\text { DTX1, FBW7, SKIP, CDK8, HIF1A, HES1, NRARP }\end{array}$ \\
\hline Total-degree & 3.94 & $\begin{array}{l}\text { NOTCH1, NOTCH2, NOTCH3, NOTCH4, GAMMA_SECRETASE, NICD1, NICD2, NICD3, NICD4, NOTCH1_PRE, NOTCH2_PRE, } \\
\text { POGLUT1, O_GLUCOSE, NOTCH3_PRE, NOTCH4_PRE, POFUT_1, O_FUCOSE, XYLE, XYL, GASE, GALACTOSE, FRINGE, } \\
\text { NGA, P53_P, NUC_NICD1, NUC_NICD2, NUC_NICD3, NUC_NICD4, CSL, COA, SMRT, COR, HDAC, CDK8, YY1, HIF1A, NRARP }\end{array}$ \\
\hline $\begin{array}{l}\text { Eigenvector } \\
\text { centrality }\end{array}$ & 0.20 & $\begin{array}{l}\text { NICD1, NICD2, NICD3, NICD4, NOTCH1_PRE, NOTCH2_PRE, NOTCH3_PRE, NOTCH4_PRE, NUC_NICD1, COA, HAT, SMRT, } \\
\text { COR, HDAC, YY1, HES1, STAT3, HES5, JAK2, HEY1, HEY2, MAG, NRARP, NFKB, MYOD, GATA3, CD44, P21, KLF5, PTCRA, } \\
\text { REL_B, C_REL, P50, P65, SOX9, BCL2, IAP, FLIP, CCND1, CCND3, MKP_1, HEYL, HES7 }\end{array}$ \\
\hline $\begin{array}{l}\text { Closeness } \\
\text { centrality }\end{array}$ & 0.002 & $\begin{array}{l}\text { JAG1, NOTCH1, JAG2, DLL1, DLL4, DLL3, NOTCH2, NOTCH3, NOTCH4, MAGP1, MAGP2, TACE, NOV, CNTN1, PRESENILIN1, } \\
\text { GAMMA_SECRETASE, APH1, NICASTRIN, PEN2, NEXT1, NEXT2, NEXT3, NEXT4, NICD1, NICD2, NICD3, NICD4, DVL, WDR12, } \\
\text { GSK_3BETA, JIP1, RAS, P53, P53_P, NUC_NICD1, NUC_NICD2, NUC_NICD3, NUC_NICD4, SMAD3, CSL, DTX1, FBW7, EP300, } \\
\text { COA, SKIP, HAT, MAML, SMRT, COR, SAP30, HDAC, CIR, SIN3A, CDK8, STAT3_P, NUC_STAT3, HIF1A, HES1, STAT3, HES5, } \\
\text { JAK2, NRARP }\end{array}$ \\
\hline $\begin{array}{l}\text { Betweenness } \\
\text { centrality }\end{array}$ & 107.94 & $\begin{array}{l}\text { NOTCH1, NOTCH2, GAMMA_SECRETASE, NEXT1, NEXT2, NEXT3, NEXT4, NICD1, NICD2, NICD3, NICD4, NUC_NICD1, NUC_ } \\
\text { NICD2, NUC_NICD3, NUC_NICD4, CSL, COA, COR, STAT3_P, NUC_STAT3, HIF1A, HES1, STAT3, HES5, NRARP, PTEN }\end{array}$ \\
\hline
\end{tabular}

Table 1: Extracted significant proteins of Notch signaling pathway which have higher parameter values than the corresponding average values.

In case of Out-Degree data, the nuclear protein CSL had highest number of Out-Degree value in the network as it is mostly connected with the output proteins of the network (more than the average value 1.97). Moreover, most of the ligands as well as the enzymes, including GAMMA_SECRETASE, and the Notch post translational modifier enzymes, such as POGLUT_1, POFUT_1, GASE, had the significant number of Out-Degree values in the network, which also signifies that activation of Notch Pathway mostly occur by the activation of these molecules in the network. On the other hand there are also some inhibitor molecules or complex, such as, Co-repressor complex (COR), HDAC, SMRT and the phosphorylated form of P53 (inhibitors of NUC_NICD1/2/3/4), which also have significant number of OutDegree values in the network. Interestingly, only HIF1A and NRARP from the output molecules of this network had significant Out-Degree and Total-Degree values, which were occurring because of the presence of feedback loops of these proteins in the network (Figure S2 and Figure S3 in Supplementary file, for Out-Degree and Total Degree values) $[63,65,80-82,85]$.

The average Eigen vector centrality of the whole network was 0.20 . We found that the nuclear transcription factor CSL had the highest value in the network. Interestingly, we also found that STAT3 had significant Eigenvector centrality in the network although it had lower number of connectivity in the network (Figure S4 in Supplementary file). In order to understand the reason behind this we found that STAT3 is connected with HES1 and HES5, which also had high Eigenvector centrality in the network. Similarly the Eigenvector centrality of NICD1/2/3/4 was also increased due to its connections with the output proteins NRARP. Therefore, it can be said that a molecule which has feedback regulations with the output proteins may increase its importance or influence in the network, even though it has lower number of connections in the network. In a reverse way, this finding helps to identify the unknown feedback interactions of a particular protein in the network. Moreover the higher value of Eigenvector centrality of transcription co-repressor protein HADC and SMRT imply their importance in the whole network.

The Closeness centrality (average 0.002) value of all individual molecules in Notch pathway revealed that CSL had the highest closeness centrality. Simultaneously, NRARP, HIF1A, STAT3 were also showing high Closeness centrality (Figure S5 in Supplementary file). Similar to the Eigen vector centrality, here also the feedback connections of these proteins with the other important proteins such as NICD1/2/3/4 or HES1/5 in the network gave the access of these proteins to regulate more number of other proteins in the network. As a result the closeness centrality values of these proteins were also increased. This result also signifies that certain perturbations or mutations of these proteins will cause worst effect than the other proteins having lower closeness centrality values.

After finding the hub or important proteins of the network on the basis of Degree, Eigenvector and Closeness centrality, we measured another kind of important centrality parameter called Betweenness centrality (Figure S6 in Supplementary file). This parameter does not assign the importance of a molecule in a signaling network on the basis of number of connections but identifies the molecules on the basis of their position (the situation of a node which lies in between the shortest path of other two nodes) in the network, higher value of which signifies higher number of signaling cascades passing through a particular node implying that all biochemical reaction cascades in general prefer the shortest route to relay the signal in much more cost effective way $[89,90]$. As expected we found that CSL had highest Betweenness centrality value in the network as the production of all the output proteins are mediated by this protein. But surprisingly we found that NICD1 had higher Betweenness centrality value compared to its other homologues (i.e., NICD2/3/4). In order to search the reason for this different result, we checked its connectivity profile and found that unlike the other three homologues of these proteins, NICD1 had extra three upstream regulators proteins: RAS, JIP1 and WDR12 as well as P53 protein in downstream. It is also connected with its nuclear counterpart NUC_NICD1, which has additional downstream target genes (e.g., BCL2, FLIP, IAP, P21, P65, P50, C_REL, REL_B) for transcription than its counterparts NUC_NICD2/3/4. Hence, more number of shortest paths intersects this protein and thus enhances the Betweenness centrality value.

Although this analysis was useful to identify the important proteins from such a large complex network and helped us to identify the probable drug targets to suppress the activity of maximum Notch target proteins, but using this simple technique we were unable to identify the exact proteins that are directly or indirectly influenced by these identified proteins, which is one of the limitations of the graph theoretical analysis. In order to overcome this drawback and to test the effect of mutation or deregulation of important proteins in the network 
under certain circumstances as well as to identify the new biomarkers of Notch pathway, we performed logical or semi-dynamic analysis of the reconstructed network.

Logical analysis of Notch signaling pathway: Logical analysis of Notch signaling network was performed to simulate the pathway activity and the expression of pathway proteins in Normal, Glioblastoma cell specific, Gamma Secreatase inhibitor treatment and two proposed drug treated scenarios, and also to see the logical relationship that exist among the proteins in the newly reconstructed Notch pathway and to analyze their regulations and expression patterns that vary according to the normal, disease and drug treated scenarios. As mentioned earlier, the highly interactive Notch pathway and its regulations and cross talks with other cancer forming pathways make this pathway more delicate and sensitive in cancer and tumor pathology. As certain mutation or change of a protein in this pathway can cause cancer, similarly without knowing the exact regulations targeting a protein in this pathway to treat cancer also can cause severe side effects, as it happens in case of Gamma Secreatase inhibition [33]. Therefore selective targeting with the knowledge of precise consequence is required and hence logical relationships/model among all the proteins in the network could be useful to identify the probable and safe drug targets.

The entire logical analysis of Notch pathway was performed using the logical relationships presented in the Table S3 as a master logical model. In the following subsections we present the outcome from the model simulations for different scenarios.

Model validation: The expression scenarios generated in the simulation for each protein in the pathway is shown in Figure 2, where GBE represents the expression of notch pathway proteins found in mRNA expression profile of Gliblastoma cell line collected from EBI-ARRAYEXPRESS database [30]. The rest of the columns (GBS, NNS, GSI, TS1 and TS2) depict the in-silico simulation results for five different types of scenarios. Figure $2 \mathrm{~A}$ presents the expression of the input proteins of our model, whereas Figure $2 \mathrm{~B}$ depicts the expression and simulation results of the intermediate and output proteins of our logical model. While comparing the simulation result of GBS with GBE, we found that there were total 54 input proteins and 62 intermediate and output proteins in our Notch pathway model.

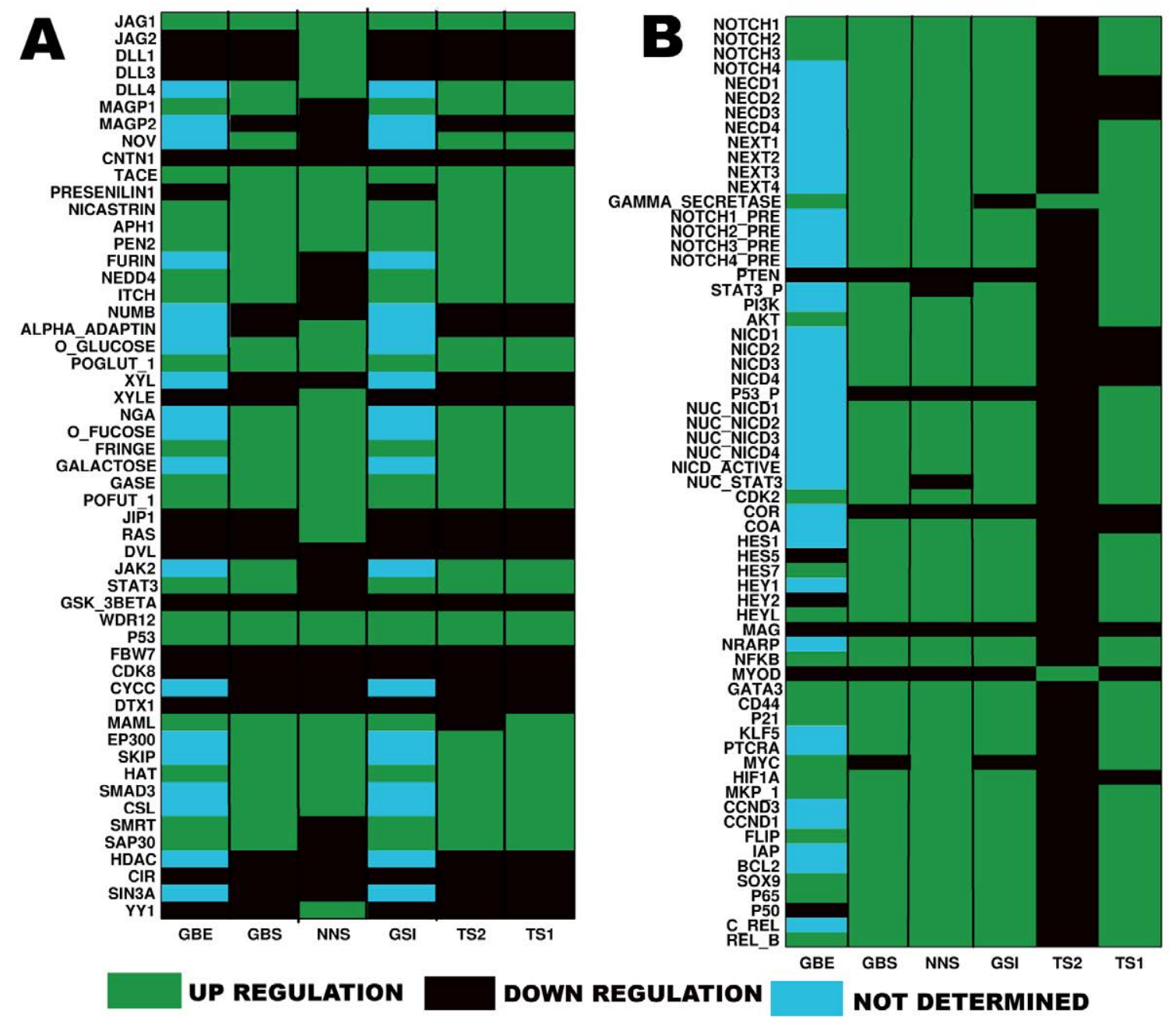

Figure 2: Expression of each protein of Notch signaling pathway in five different scenarios. mRNA expression profile of Gliblastoma Cell Line (GBE) [30], Glioblastoma (GBS), Normal Notch Pathway (NNS), Gamma Secreatase Inhibition (GSI), and two proposed drug treated scenarios; TS2: NICD1 and MAML combinatorial inhibition, and TS1: NICD1 and HIF1A combinatorial inhibition. (A) The expression of the input proteins (B) The expression and simulation results of the intermediate and output proteins 
Out of the total 54 input molecules, the exact expression level (UP or DOWN) were found for 35 proteins, whereas expressions of rest of the 19 proteins were found "Not determined". The expressions levels of these 19 molecules were collected from published experimental results of Glioblastoma cell line [91-104]. On the other hand, in the output and intermediate proteins out of 62 proteins, we found the expressions of 25 proteins from mRNA expression data of Glioblastoma cell line. Our simulation (GBS) was able to correctly predict the expression of 21 molecules with an accuracy of $84 \%$. Our simulation was able to predict the probable expressions of the remaining 38 proteins, which were also experimentally verified.

Model validation for Gamma Secreatase inhibition: GAMMA SECREATASE, which is one of the main activator proteins of Notch pathway, has been used as a drug target for several cancers or tumor treatment experiments so that excess Notch pathway activity could be blocked. Also the feasibility of targeting/ inhibiting this membrane associated metallo-protease enzyme makes it as a common choice for all clinical and experimental biologists $[9,32]$.

GAMMA SECRETASE inhibited Glioblastoma cell model (GSI) was created and simulated by considering the logical state of this protein as " 0 " or 'OFF' in our Glioblastoma cell model scenario (GBS). We observed that after inhibiting the GAMMA SECRETASE enzyme in Glioblastoma cell line, the number of upstream activator and inhibitor molecules were reducing significantly as compared to the GBS (Figure 3A and Figure 3C). In order to validate this simulation result with experimental data, we considered the previous experimental findings of DAPT, BMS-708163 and RO4929097 (known GAMMA SECRETASE inhibitors) treated expressions profile of Notch pathway proteins in Glioblastoma cell line [105]. It shows that around 17 genes including the Notch pathway genes such as notch1, notch3, hes1, maml, dll3, jag2, etc., were found active in the non-responder GAMMA SECRETASE inhibited cell populations as compared to the inhibitor responded cell populations. Same result is also found from our in-silico simulation of GAMMA SECRETASE Inhibitor scenario (GSI) by comparing the number of upstream activators of the above mentioned genes/ proteins in GBS and GSI scenarios (Figure 3A). Simultaneously we also observed from the simulation result that the downstream activated proteins of several Notch pathway activator proteins (e.g., JAG1/2, DLL1/3/4, MAGP1, NICD1 etc.)) were getting reduced by administering the GAMMA SECRETASE inhibition in GBS cell line (Figure 3B). These results clearly validate our logical model for GBS and GSI scenarios with the experimental results obtained from previously done cancer stem cell and molecular biology experiments [105].

Comparison between Normal and Glioblastoma scenario: In order to observe the change of the expressions of different Notch pathway proteins in Normal (NNS) and Glioblastoma scenarios (GBS), we simulated our model for both the scenarios and compared to extract and identify the pathway molecules that were showing significant fluctuations in both scenarios. The logical states of the input proteins were considered as same as shown in Figure 2 and the expression levels are provided in Table S4 and Table S5 of Supplementary file. By calculating the dependency matrices for both the scenarios, we were able to identify significant variations of Upstream Activators, Upstream Inhibitors, Downstream Activated and Inhibited proteins for the proteins reported in the $\mathrm{X}$-axis of Figure 3.

Here, the simulation result of Normal Notch pathway scenario (NNS) served as a control to measure the change in the expression level of Notch pathway molecules in Glioblastoma scenario. In this analysis we correlated the expression level of a protein with its total number of Upstream Activators and Inhibitors molecules. We found that different types of proteins from different sub-cellular locations were showing significant changes (Figure 3A and Figure 3C) in Glioblastoma scenario (GBS) compared to the Normal Notch pathway scenario (NNS). Considering the fact that the expression of a protein in a signaling network is directly proportional to its number of upstream activator molecules and inversely proportional to its upstream inhibitor molecules [39], we were able to extract the proteins which were causing Glioblastoma by mutating the Notch signal and its associated molecules. Mostly all the Notch target proteins (Oncoproteins of Glioblastoma such as MAG, BCL2, MYOD etc.), of GBS had higher number of upstream activators as compared to the NNS (Figure 3C). On the other hand the total number of inhibitor molecules (Upstream inhibitor) acting on the Notch target proteins of NNS scenario was comparably higher than the GBS scenario. Higher the inhibition by upstream inhibitors on Notch target proteins, lower their expressions and thus controls several phenotypic expressions such as cell proliferation, myelination, anti-apoptosis etc.,. This meticulous regulation gets perturbed by several oncogenic factors in Glioblastoma scenario and the inhibitions on the output proteins are withdrawn [2224,63]. The number of downstream activated proteins of Notch pathway (GAMMA_SECRETASE, WDR12, NICD1, NICD4, EP300, MAML, SKIP, HAT etc.,) was also showing the variations as compared to the NNS (Figure 3B). It was also revealed that the downstream activator molecules of HES1, HES5 and HIF1A were increased from 0 to around 50 in GBS scenario. Surely this finding gave us a preliminary direction to identify the probable drug targets of Glioblastoma treatment. It is also worth mentioning that all these above mentioned molecules are the transcription activators or co-activators of Notch pathway and their up-regulation may be the main reason to increase the activations/ productions of Notch target proteins in GBS. However, the number of downstream inhibited molecules of most of the molecules were not showing significant variations in all the five scenarios (Figure 3D), except for NUC_NCD1, FBW7, CDK8, COR and HEY1. Moreover, there was no significant variation in the number of downstream inhibited molecules for GBS, NNS and GSI scenarios.

In-silico identification of alternative drug treated scenarios: In order to find out the alternative drug targets, in place of GAMMA SECRETASE inhibitors/ drugs to treat Glioblastoma tumor cell line, we examined several sole and combinatorial targets (mostly proteins) of our Notch pathway model. Identification of such combinations was not easier, especially when the numbers of probable options were very high in such a large signaling network. Selection of the target molecules from a signaling network depends on its topological structure (i.e., connectivity, centrality) as well its logical relationship with all other molecules in the network. From our graph theoretical analysis we were able to identify few important proteins like ADAM/ TACE, CSL, NICD1, MAML, HIF1A, NRARP, HES1, HES5 etc., which had high centrality values within the network (Table 1). Furthermore, on the basis of the biological feasibility and the evidence of being used as targets in previous experiments, we were able to filter out the proteins like ADAM/TACE, NICD1, MAML, HIF1A, DLL4, as probable drug targets for our analysis. It should be noted that almost all these identified proteins also show the variations of high expression level in GBS Scenario (Figure 3C). Thus by integrating the results from network, logical and reported experimental results, our in-silico modeling approach was able to extract the possible combinations of few proteins to treat Glioblastoma scenario. This finding was useful to bring down the number of probable drug targets from more than 100 proteins of the network to 4 or 5 proteins. 

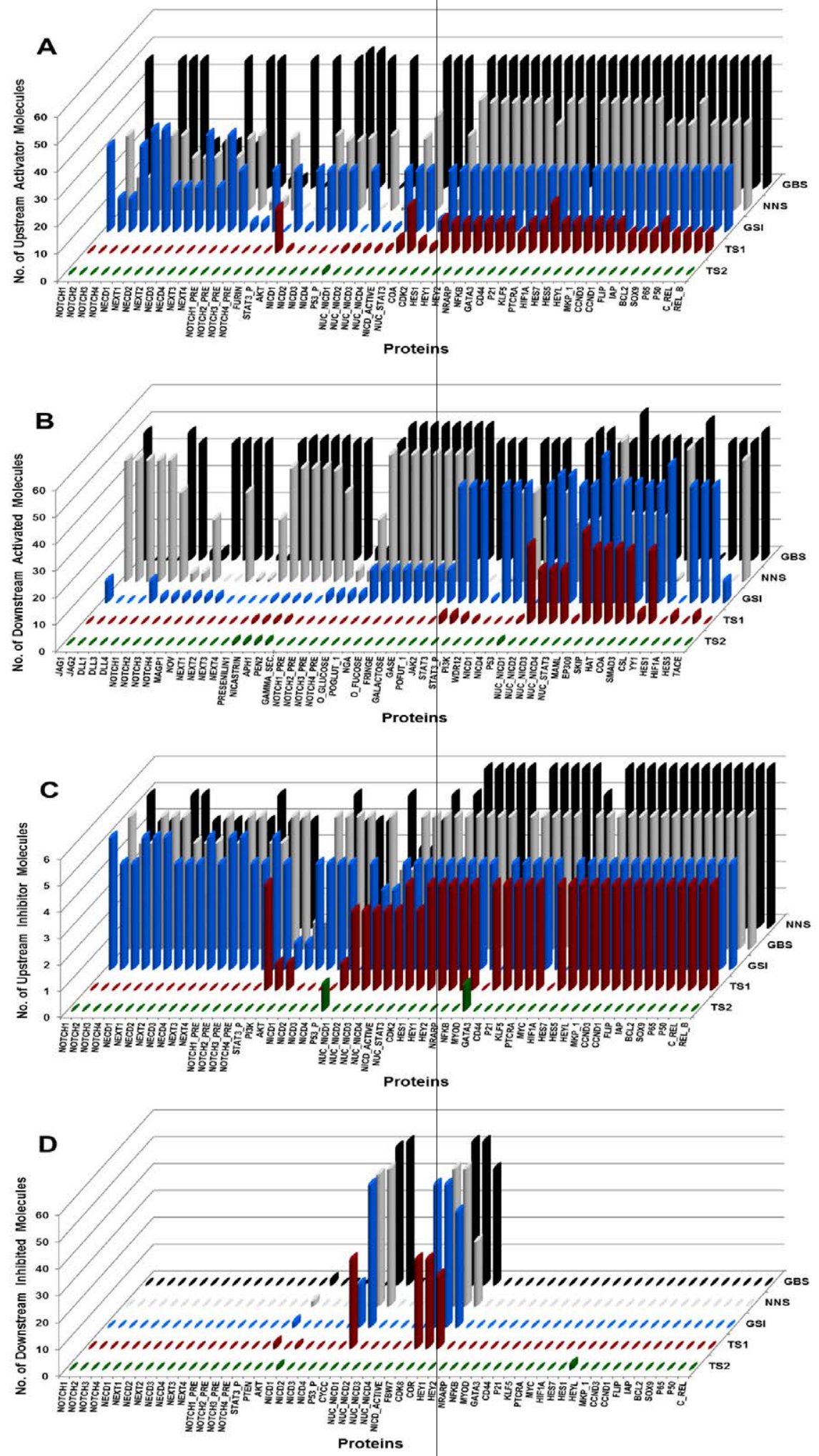

Figure 3: Comparison between normal, glioblastoma, gamma secretase inhibition and two proposed drug target scenarios. NNS: Normal Notch Pathway; GBS: Glioblastoma Scenario; GSI: Gamma Secretase Inhibition; TS1: NICD1 and HIF1A combinatorial inhibition; TS2: NICD1 and MAML combinatorial inhibition. (A) Represents number of upstream activator molecules ( $Y$-axis) activating the molecules (X-axis) representing significant variations (B) Represents number of downstream activated molecules (Y-axis) activated by the molecules (X-axis) representing significant variations (C) Represents number of upstream inhibitor molecules (Y-axis) inhibiting the molecules (X-axis) representing significant variations (D) Represents number of downstream inhibited molecules (Y-axis) inhibited by the molecules (X-axis) representing significant variations. 
Using these identified lead targets, we further targeted each protein as a sole target, but unfortunately none of the target gave significant result (result not shown). Eventually, we had to look for the combinations of the proteins from all the lead target molecules. We observed that the synergistic effect of targeting the multiple proteins in combinations was giving good result as compared to the results we were getting from the individual in-silico targeting. We tried several possible combinations while doing this in-silico drug treated perturbation analysis and the best results found from that analysis was presented in TS1 and TS2 scenarios of Figure 3. TS1 represents NICD1 and HIF1A combinatorial drug treated scenario whereas TS2 represents the NICD1 and MAML treated scenario. In TS1 scenario, we were able to suppress partially but comparably lower expressions of Notch target onco-proteins (BCL2, HES1, MAG, IAP etc.,) as compared to Glioblastoma as well as GAMMA SECRETASE Inhibitor scenario (GSI). On the other hand, in TS2 scenario, the expressions of the target onco-proteins were completely suppressed (Figure 3 ). Thus, both the partial inhibition and complete suppression can be achieved by using TS1 and TS2 scenarios, respectively.

\section{Discussion}

The involvement of the Notch signaling pathway in cancer stem cell generations have been reported in many molecular and cancer stem cell experiments [1-3]. Inhibition of this pathway by several drug targets in various tumor cell lines including Glioblastoma, Oligodendrocyte growth is also been tested $[32,35]$. Synthesis of several small molecule drugs or inhibitors of this pathway prove the significance and importance of this pathway to the pharmaceutical experiments of cancer pathology. Unfortunately despite several attempts to suppress the cancer progression by inhibiting the molecules of this pathway, the fruitful results are yet to come. Recently developed several GAMMA SECRETASE inhibitors were also found to have several toxic effects $[33,34]$. Hence a better understanding of the complete map of Notch signaling is useful before developing any drug targets against cancer, as it is a highly regulated as well as sensitive signaling pathway cross connected with several other signaling and metabolic pathways in cell. Unfortunately, there is no complete map available which can give information about its regulations with other cross talking molecules. Therefore in our work, we first reconstructed the entire Notch signaling map by curating the data from all the available resources especially from literatures and signaling database. In this analysis, we reconstructed a new notch signaling network with 115 molecules (including protein complex, metabolites etc.) and 231 reactions (e.g., Phosphorylation, physical interactions, proteolytic cleavage etc.,). To the best of our knowledge this is largest human cell specific Notch signaling map. This pathway and its associated molecules can also be used as biomarkers for the detection of cancer and the identification of drug targets for further in-vitro and in-vivo analysis.

Using this reconstructed network, we further analyzed structurally the network topology by graph theoretical model and were able to identify the centrally located proteins forming the "Hub" in the network. We found that the nuclear transcription factor CSL, STAT3 had the higher eigenvector centrality value in the network along with HES1 and HES5. Similarly the Eigenvector centrality of NICD1/2/3/4 was also increased due to its connections with the output proteins NRARP, implying that these proteins not only have high number of connections but also are connected with other highly prestigious nodes that possess higher number of connections in the network. We were also able to identify the connection of several cross talking molecules, such as, HIF1A from Hypoxia, PTEN from PI3K/AKT pathway, P53,
RAS etc., with the core molecules of Notch pathway and creation of either positive or negative feedback loops in the network. Moreover, CSL, NRARP, HIF1A, STAT3 were also showing high Closeness centrality, where the feedback connections of these proteins with the other important proteins such as NICD1/2/3/4 or HES1/5 in the network gave the access of these proteins to regulate more number of other proteins in the network, resulting in an increased closeness centrality values of these proteins. This also signifies that certain perturbations or mutations of these proteins will cause worst effect than the other proteins having lower closeness centrality value. The higher Betweenness centrality value found for NICD1 compare to its other homologues (i.e., NICD2/3/4) shows that unlike the other three homologues of these proteins, NICD1 had extra three upstream regulators proteins: RAS, JIP1 and WDR12 as well as P53 protein in downstream. It is also connected with its nuclear counterpart NUC_NICD1, which has additional downstream target genes (e.g., BCL2, FLIP, IAP, P21, P65, P50, C_REL, REL_B) for transcription than its counterparts NUC_NICD2/3/4, implying enhancement of Betweenness centrality value as more numbers of shortest paths intersect this protein. This structural and topological analysis helps us to identify the probable drug targets to suppress the activity of maximum Notch target proteins.

For facilitating clinical and experimental pharmacologists to perform further in-vivo and in-vitro experiments in real cancer cell model and to test the effect of mutation or deregulation of important proteins in the network under certain circumstances as well as to identify the new biomarkers of Notch pathway, we followed a semidynamic computational approach, logical analysis. In the logical analysis we modeled the entire pathway reactions by logical equations and created five scenarios: Normal (NNS), Glioblastoma (GBS), GAMMA SECRETASE inhibition (GSI), Treatment scenarios by inhibiting NICD1 and HIF1A (TS1) and by inhibiting NICD1 and MAML (TS2). We also validated our model with the mRNA expression levels measured in human Glioblastoma cell line [30]. Further we were also able to verify the expression or activation of GSI with the reported experimental findings [105]. From our in-silico simulation of GSI by comparing the number of upstream activators genes/ proteins in GBS and GSI scenarios, we observed that the downstream activated proteins of several Notch pathway activator proteins (e.g., JAG1/2, DLL1/3/4, MAGP1, NICD1 etc.,) were getting reduced by administering the GAMMA SECRETASE inhibition in GBS cell line, which not only prove the reported experimental findings [105] but also validate our computational study.

Moreover, by comparing the NNS and GBS, we were also able to identify the proteins which were abnormally getting activated or inhibited in Glioblastoma cell line compared to the normal scenario. Along with our network analysis, these findings gave us the opportunity to filter out the possible drug target molecules from out of 115 molecules of the pathway. We identified several probable targets through sole or combinations of proteins by perturbing the logical states of GBS model. Though the sole perturbation did not show promising results, but targeting these proteins in combinations showed promising result in suppressing the expressions of several Notch target proteins. The synergistic effect imposed by using the combinatorial drug targets has improved the result in several folds. Among these possible combinations we observed NICD1 and HIF1A (TS1) are suitable for the partial blocking of Notch pathway activity whereas inhibition of NICD1 and MAML (TS2) is useful to completely suppress the pathway activity. It can also be concluded that depending 
on the critical situations of Glioblastoma, one can use any of the above combinations to suppress the growth of Glioblastoma.

Through our reconstruction and computational study of the human cell specific Notch signaling pathway we get an insight and complete understanding of the interactions between the signaling proteins in the pathway along with identification of alternative drug targets for Glioblastoma, where the pathway is known to become mutated. The computational strategy and alternative drug targets predicted from our analysis may help to achieve more accurate therapeutic strategy, not only for Glioblastoma but also for other diseased conditions. From our analysis the perturbation effects of minimal combination of proteins and identification of new combinatorial drug targets or pathway signatures provide a more sensible strategy for finding therapeutic targets for cancer. Our findings will be useful for the experimental and clinical pharmacologists to select the biomarkers for cancer prognosis and will show new direction in human cell specific signaling pathway study.

\section{Acknowledgement}

We thank Mr. Shomeek Chowdhury, Summer Research Fellow, Indian Academy of Sciences, for collating few data for pathway reconstruction. We also thank The Director, CSIR-National Chemical Laboratory, Pune for providing the required infrastructures related to this work under CSIR-NCL grant MLP026226. The work is funded by the Department of Biotechnology, Govt. of India, Grant No. BT/PR13689/BID/07/363/2010.

\section{References}

1. Oh P, Lobry C, Gao J, Tikhonova A, Loizou E, et al. (2013) In vivo mapping of notch pathway activity in normal and stress hematopoiesis. Cell Stem Cell 13: $190-204$

2. Gridley $\mathrm{T}$ (2007) Notch signaling in vascular development and physiology. Development 134: 2709-2718.

3. Artavanis-Tsakonas S, Rand MD, Lake RJ (1999) Notch signaling: cell fate control and signal integration in development. Science 284: 770-776.

4. Nagao M, Sugimori M, Nakafuku M (2007) Cross talk between notch and growth factor/cytokine signaling pathways in neural stem cells. Mol Cell Biol 27: 3982-3994.

5. Guo S, Gonzalez-Perez RR (2011) Notch, IL-1 and leptin crosstalk outcome (NILCO) is critical for leptin-induced proliferation, migration and VEGF/ VEGFR-2 expression in breast cancer. PLoS One 6: e21467.

6. Ables JL, Breunig JJ, Eisch AJ, Rakic P (2011) Not(ch) just development: Notch signalling in the adult brain. Nat Rev Neurosci 12: 269-283.

7. Brou C, Logeat F, Gupta N, Bessia C, LeBail O, et al. (2000) A novel proteolytic cleavage involved in Notch signaling: the role of the disintegrin-metalloprotease TACE. Mol Cell 5: 207-216.

8. Fortini ME (2002) Gamma-secretase-mediated proteolysis in cell-surfacereceptor signalling. Nat Rev Mol Cell Biol 3: 673-684.

9. Shao H, Huang Q, Liu ZJ (2012) Targeting Notch signaling for cancer therapeutic intervention. Adv Pharmacol 65: 191-234.

10. Blokzijl A, Dahlqvist C, Reissmann E, Falk A, Moliner A, et al. (2003) Cross-talk between the Notch and TGF-beta signaling pathways mediated by interaction of the Notch intracellular domain with Smad3. J Cell Biol 163: 723-728.

11. Zhou S, Hayward SD (2001) Nuclear localization of CBF1 is regulated by interactions with the SMRT corepressor complex. Mol Cell Biol 21: 6222-6232.

12. Hsieh JJ, Zhou S, Chen L, Young DB, Hayward SD (1999) CIR, a corepressor linking the DNA binding factor CBF1 to the histone deacetylase complex. Proc Natl Acad Sci U S A 96: 23-28.

13. Zhou S, Fujimuro M, Hsieh JJ, Chen L, Miyamoto A, et al. (2000) SKIP, a CBF1-associated protein, interacts with the ankyrin repeat domain of NotchIC To facilitate NotchIC function. Mol Cell Biol 20: 2400-2410.

14. Fang TC, Yashiro-Ohtani Y, Del Bianco C, Knoblock DM, Blacklow SC, et al. (2007) Notch directly regulates Gata3 expression during T helper 2 cell differentiation. Immunity 27: 100-110.
15. Katoh M, Katoh M (2006) Notch ligand, JAG1, is evolutionarily conserved target of canonical WNT signaling pathway in progenitor cells. Int J Mol Med 17: 681 685

16. Joshi I, Minter LM, Telfer J, Demarest RM, Capobianco AJ, et al. (2009) Notch signaling mediates $\mathrm{G} 1 / \mathrm{S}$ cell-cycle progression in T cells via cyclin D3 and its dependent kinases. Blood 113: 1689-1698.

17. Ronchini C, Capobianco AJ (2001) Induction of cyclin D1 transcription and CDK2 activity by Notch(ic): implication for cell cycle disruption in transformation by Notch(ic). Mol Cell Biol 21: 5925-5934.

18. Rodilla V, Villanueva A, Obrador-Hevia A, Robert-Moreno A, FernándezMajada V, et al. (2009) Jagged1 is the pathological link between Wnt and Notch pathways in colorectal cancer. Proc Natl Acad Sci U S A 106: 6315-6320.

19. Liu H, Chi AW, Arnett KL, Chiang MY, Xu L, et al. (2010) Notch dimerization is required for leukemogenesis and T-cell development. Genes Dev 24: 23952407.

20. Kondoh K, Sunadome K, Nishida E (2007) Notch signaling suppresses p38 MAPK activity via induction of MKP-1 in myogenesis. J Biol Chem 282: $3058-$ 3065.

21. Ang HL, Tergaonkar $V$ (2007) Notch and NFkappaB signaling pathways: Do they collaborate in normal vertebrate brain development and function? Bioessays 29: 1039-1047.

22. Sade H, Krishna S, Sarin A (2004) The anti-apoptotic effect of Notch-1 requires p56lck-dependent, Akt/PKB-mediated signaling in T cells. J Biol Chem 279 2937-2944.

23. Cheng P, Zlobin A, Volgina V, Gottipati S, Osborne B, et al. (2001) Notch-1 regulates NF-kappaB activity in hemopoietic progenitor cells. J Immunol 167 4458-4467.

24. Rangarajan A, Talora C, Okuyama R, Nicolas M, Mammucari C, et al. (2001) Notch signaling is a direct determinant of keratinocyte growth arrest and entry into differentiation. EMBO J 20: 3427-3436.

25. Espinoza I, Miele L (2013) Deadly crosstalk: Notch signaling at the intersection of EMT and cancer stem cells. Cancer Lett

26. Hernandez SL, Banerjee D, Garcia A, Kangsamaksin T, Cheng WY, et al (2013) Notch and VEGF pathways play distinct but complementary roles in tumor angiogenesis. Vasc Cell 5: 17.

27. Koizumi Y, Iwasa Y, Hirashima T (2012) Mathematical study of the role of Delta/Notch lateral inhibition during primary branching of Drosophila trachea development. Biophys J 103: 2549-2559.

28. Li JL, Sainson RC, Shi W, Leek R, Harrington LS, et al. (2007) Delta-like 4 Notch ligand regulates tumor angiogenesis, improves tumor vascular function, and promotes tumor growth in vivo. Cancer Res 67: 11244-11253.

29. Asnaghi L, Handa JT, Merbs SL, Harbour JW, Eberhart CG (2013) A role for Jag2 in promoting uveal melanoma dissemination and growth. Invest Ophthalmol Vis Sci 54: 295-306.

30. Sun L, Hui AM, Su Q, Vortmeyer A, Kotliarov Y, et al. (2006) Neuronal and glioma-derived stem cell factor induces angiogenesis within the brain. Cancer Cell 9: 287-300.

31. Purow B (2012) Notch inhibition as a promising new approach to cance therapy. Adv Exp Med Biol 727: 305-319.

32. Zou Y, Cao Y, Yue Z, Liu J (2012) Gamma-secretase inhibitor DAPT suppresses glioblastoma growth via uncoupling of tumor vessel density from vessel function. Clin Exp Med.

33. Doody RS, Raman R, Farlow M, Iwatsubo T, Vellas B, et al. (2013) A phase 3 trial of semagacestat for treatment of Alzheimer's disease. N Engl J Med 369: 341-350.

34. Osherovich L (2011) Notch nicked again. SciBX 4 .

35. Floyd DH, Kefas B, Seleverstov O, Mykhaylyk O, Dominguez C, et al. (2012) Alpha-secretase inhibition reduces human glioblastoma stem cell growth in vitro and in vivo by inhibiting Notch. Neuro Oncol 14: 1215-1226.

36. Banerjee S, Sarkar RR (2008) Delay-induced model for tumor-immune interaction and control of malignant tumor growth. Biosystems 91: 268-288.

37. Sherriff MR, Sarkar RR (2008) Computational approaches and modeling of signaling processes in the Immune System. Proc Indian Natn Sci Acad 74:187200. 
38. Morris MK, Saez-Rodriguez J, Sorger PK, Lauffenburger DA (2010) Logicbased models for the analysis of cell signaling networks. Biochemistry 49: 3216-3224.

39. Chowdhury S, Pradhan RN, Sarkar RR (2013) Structural and logical analysis of a comprehensive hedgehog signaling pathway to identify alternative drug targets for glioma, colon and pancreatic cancer. PLoS One 8: e69132.

40. Wagner A, Fell DA (2001) The small world inside large metabolic networks Proc Biol Sci 268: 1803-1810.

41. Samaga R, Saez-Rodriguez J, Alexopoulos LG, Sorger PK, Klamt S (2009) The logic of EGFR/ErbB signaling: theoretical properties and analysis of highthroughput data. PLoS Comput Biol 5: e1000438.

42. Klamt S, Saez-Rodriguez J, Lindquist JA, Simeoni L, Gilles ED (2006) A methodology for the structural and functional analysis of signaling and regulatory networks. BMC Bioinformatics 7: 56 .

43. Wang HY, Huang YX, Qi YF, Zhang Y, Bao YL, et al. (2013) Mathematical models for the Notch and Wnt signaling pathways and the crosstalk between them during somitogenesis. Theor Biol Med Model 10: 27

44. Terry AJ, Sturrock M, Dale JK, Maroto M, Chaplain MA (2011) A spatio-temporal model of Notch signalling in the zebrafish segmentation clock: conditions for synchronised oscillatory dynamics. PLoS One 6: e16980.

45. Srividhya J (2012) A Mathematical Model for Inter-Cellular Inductive Notch Signaling. Computational Molecular Bioscience 2: 102-107.

46. Kitano H, Funahashi A, Matsuoka Y, Oda K (2005) Using process diagrams for the graphical representation of biological networks. Nat Biotechnol 23: 961 966

47. Funahashi A, Tanimura N, Morohashi M, Kitano H (2003) CellDesigner: a process diagram editor for gene-regulatory and biochemical networks. BIOSILICO 1:159-162.

48. Al-Hussaini H, Subramanyam D, Reedijk M, Sridhar SS (2011) Notch signaling pathway as a therapeutic target in breast cancer. Mol Cancer Ther 10: 9-15.

49. Tagami S, Okochi M, Yanagida K, Ikuta A, Fukumori A, et al. (2008) Regulation of Notch signaling by dynamic changes in the precision of S3 cleavage of Notch-1. Mol Cell Biol 28: 165-176.

50. Houde C, Li Y, Song L, Barton K, Zhang Q, et al. (2004) Overexpression of the NOTCH ligand JAG2 in malignant plasma cells from multiple myeloma patients and cell lines. Blood 104: 3697-3704.

51. Fassler M, Li X, Kaether C (2011) Polar transmembrane-based amino acids in presenilin 1 are involved in endoplasmic reticulum localization, Pen2 protein binding, and $\gamma$-secretase complex stabilization. J Biol Chem 286: 38390-38396.

52. Miyamoto A, Lau R, Hein PW, Shipley JM, Weinmaster G (2006) Microfibrillar proteins MAGP-1 and MAGP-2 induce Notch1 extracellular domain dissociation and receptor activation. J Biol Chem 281: 10089-10097.

53. Sakamoto K, Yamaguchi S, Ando R, Miyawaki A, Kabasawa Y, et al. (2002) The nephroblastoma overexpressed gene (NOV/ccn3) protein associates with Notch1 extracellular domain and inhibits myoblast differentiation via Notch signaling pathway. J Biol Chem 277: 29399-29405.

54. Hu QD, Ang BT, Karsak M, Hu WP, Cui XY, et al. (2003) F3/contactin acts as a functional ligand for Notch during oligodendrocyte maturation. Cell 115 : 163-175

55. Hu QD, Ma QH, Gennarini G, Xiao ZC (2006) Cross-talk between F3/contactin and Notch at axoglial interface: a role in oligodendrocyte development. Dev Neurosci 28: 25-33.

56. Gordon WR, Vardar-Ulu D, L'Heureux S, Ashworth T, Malecki MJ, et al. (2009) Effects of S1 cleavage on the structure, surface export, and signaling activity of human Notch1 and Notch2. PLoS One 4: e6613.

57. Logeat F, Bessia C, Brou C, LeBail O, Jarriault S, et al. (1998) The Notch1 receptor is cleaved constitutively by a furin-like convertase. Proc Natl Acad Sci U S A 95: 8108-8112.

58. Kadesch T (2004) Notch signaling: the demise of elegant simplicity. Curr Opin Genet Dev 14: 506-512.

59. Beres BJ, George R, Lougher EJ, Barton M, Verrelli BC, et al. (2011) Numb regulates Notch1, but not Notch3, during myogenesis. Mech Dev 128: 247-257.

60. Nagpal P, Plant PJ, Correa J, Bain A, Takeda M, et al. (2012) The ubiquitin ligase Nedd4-1 participates in denervation-induced skeletal muscle atrophy in mice. PLoS One 7: e46427.
61. Koncarevic A, Jackman RW, Kandarian SC (2007) The ubiquitin-protein ligase Nedd4 targets Notch1 in skeletal muscle and distinguishes the subset of atrophies caused by reduced muscle tension. FASEB J 21: 427-437.

62. Cotter D, Honavar M, Lovestone S, Raymond L, Kerwin R, et al. (1999) Disturbance of Notch-1 and Wnt signalling proteins in neuroglial balloon cells and abnormal large neurons in focal cortical dysplasia in human cortex. Acta Neuropathol 98: 465-472.

63. Kim MY, Ann EJ, Mo JS, Dajas-Bailador F, Seo MS, et al. (2010) JIP1 binding to RBP-Jk mediates cross-talk between the Notch1 and JIP1-JNK signaling pathway. Cell Death Differ 17: 1728-1738.

64. Mittal S, Subramanyam D, Dey D, Kumar RV, Rangarajan A (2009) Cooperation of Notch and Ras/MAPK signaling pathways in human breast carcinogenesis. Mol Cancer 8: 128.

65. Natarajan S, Li Y, Miller EE, Shih DJ, Taylor MD, et al. (2013) Notch1-induced brain tumor models the sonic hedgehog subgroup of human medulloblastoma. Cancer Res 73: 5381-5390

66. Tateya T, Imayoshi I, Tateya I, Ito J, Kageyama R (2011) Cooperative functions of Hes/Hey genes in auditory hair cell and supporting cell development. Dev Biol 352: 329-340.

67. Zheng X, Linke S, Dias JM, Zheng X, Gradin K, et al. (2008) Interaction with factor inhibiting HIF-1 defines an additional mode of cross-coupling between the Notch and hypoxia signaling pathways. Proc Natl Acad Sci U S A 105 3368-3373.

68. Kuroda K, Tani S, Tamura K, Minoguchi S, Kurooka H, et al. (1999) Deltainduced Notch signaling mediated by RBP-J inhibits MyoD expression and myogenesis. J Biol Chem 274: 7238-7244.

69. Bastian M, Heymann S, Jacomy M (2009) Gephi: an open source software for exploring and manipulating networks. International AAAl Conference on Weblogs and Social Media.

70. Csardi G, Nepusz T (2006) The igraph software package for complex network research. InterJournal, Complex Systems 1695.

71. Klamt S, Saez-Rodriguez J, Gilles ED (2007) Structural and functional analysis of cellular networks with CellNetAnalyzer. BMC Syst Biol 1: 2

72. Klamt S, von Kamp A (2011) An application programming interface for CellNetAnalyzer. Biosystems 105: 162-168.

73. Han X, Ju JH, Shin I (2012) Glycogen synthase kinase 3-î phosphorylates novel S/T-P-S/T domains in Notch1 intracellular domain and induces its nuclear localization. Biochem Biophys Res Commun 423: 282-288.

74. Collu GM, Hidalgo-Sastre A, Acar A, Bayston L, Gildea C, et al. (2012) Dishevelled limits Notch signalling through inhibition of CSL. Development 139: 4405-4415.

75. Lee TV, Sethi MK, Leonardi J, Rana NA, Buettner FF, et al. (2013) Negative regulation of notch signaling by xylose. PLoS Genet 9: e1003547.

76. Brückner K, Perez L, Clausen H, Cohen S (2000) Glycosyltransferase activity of Fringe modulates Notch-Delta interactions. Nature 406: 411-415.

77. Yamakawa T, Ayukawa T, Matsuno K (2012) Metabolism and transportation pathways of GDP-fucose that are required for the O-fucosylation of Notch. Adv Exp Med Biol 727: 37-46.

78. Haltiwanger RS, Stanley P (2002) Modulation of receptor signaling by glycosylation: fringe is an O-fucose-beta1,3-N-acetylglucosaminyltransferase. Biochim Biophys Acta 1573: 328-335.

79. Kamakura S, Oishi K, Yoshimatsu T, Nakafuku M, Masuyama N, et al. (2004) Hes binding to STAT3 mediates crosstalk between Notch and JAK-STAT signalling. Nat Cell Biol 6: 547-554.

80. Chen L, Zhang R, Li P, Liu Y, Qin K, et al. (2013) P53-induced microRNA-107 inhibits proliferation of glioma cells and down-regulates the expression of CDK6 and Notch-2. Neurosci Lett 534: 327-332.

81. Giovannini C, Gramantieri L, Chieco P, Minguzzi M, Lago F, et al. (2009) Selective ablation of Notch3 in HCC enhances doxorubicin's death promoting effect by a p53 dependent mechanism. J Hepatol 50: 969-979.

82. Sun Y, Klauzinska M, Lake RJ, Lee JM, Santopietro S, et al. (2011) Trp53 regulates Notch 4 signaling through Mdm2. J Cell Sci 124: 1067-1076.

83. Pawlus MR, Wang L, Hu CJ (2013) STAT3 and HIF1 1 \pm cooperatively activate HIF1 target genes in MDA-MB-231 and RCC4 cells. Oncogene. 
Citation: Chowdhury S, Sarkar RR (2013) Drug Targets and Biomarker Identification from Computational Study of Human Notch Signaling Pathway. Clin Exp Pharmacol 3: 137. doi:10.4172/2161-1459.1000137

84. Palomero T, Dominguez M, Ferrando AA (2008) The role of the PTEN/AKT Pathway in NOTCH1-induced leukemia. Cell Cycle 7: 965-970.

85. Pirot P, van Grunsven LA, Marine JC, Huylebroeck D, Bellefroid EJ (2004) Direct regulation of the Nrarp gene promoter by the Notch signaling pathway. Biochem Biophys Res Commun 322: 526-534.

86. Costenbader E, Valente TW (2003) The stability of centrality measures when networks are sampled. Social Networks 25: 283-307.

87. Ozgür A, Vu T, Erkan G, Radev DR (2008) Identifying gene-disease associations using centrality on a literature mined gene-interaction network. Bioinformatics 24: i277-285.

88. Di Deco J, González AM, Díaz J, Mato V, García-Frank D, et al. (2013) Machine learning and social network analysis applied to Alzheimer's disease biomarkers. Curr Top Med Chem 13: 652-662.

89. Sridharan GV, Hassoun S, Lee K (2011) Identification of biochemical network modules based on shortest retroactive distances. PLoS Comput Biol 7: e1002262.

90. Honjo T (1996) The shortest path from the surface to the nucleus: RBP-J kappa/Su(H) transcription factor. Genes Cells 1: 1-9.

91. El Hindy N, Keyvani K, Pagenstecher A, Dammann P, Sandalcioglu IE, et al. (2013) Implications of DIl4-Notch signaling activation in primary glioblastoma multiforme. Neuro Oncol 15: 1366-1378.

92. Laurent M, Martinerie C, Thibout H, Hoffman MP, Verrecchia F, et al. (2003) NOVH increases MMP3 expression and cell migration in glioblastoma cells via a PDGFR-alpha-dependent mechanism. FASEB J 17: 1919-1921.

93. Leitlein J, Aulwurm S, Waltereit R, Naumann U, Wagenknecht B, et al. (2001) Processing of immunosuppressive pro-TGF-beta 1,2 by human glioblastoma cells involves cytoplasmic and secreted furin-like proteases. J Immunol 166: 7238-7243.

94. Jiang $X$, Xing $H$, Kim TM, Jung $Y$, Huang $W$, et al. (2012) Numb regulates glioma stem cell fate and growth by altering epidermal growth factor receptor and Skp1-Cullin-F-box ubiquitin ligase activity. Stem Cells 30: 1313-1326.

95. Tucker-Burden C, Chappa P, Krishnamoorthy M, Gerwe BA, Scharer CD, et al. (2012) Lectins identify glycan biomarkers on glioblastoma-derived cancer stem cells. Stem Cells Dev 21: 2374-2386.
96. Tsuchiya N, Yamanaka R, Yajima N, Homma J, Sano M, et al. (2005) Isolation and characterization of an $\mathrm{N}$-linked oligosaccharide that is increased in glioblastoma tissue and cell lines. Int J Oncol 27: 1231-1239.

97. Kushchayev SV, Sankar T, Eggink LL, Kushchayeva YS, Wiener PC, et al (2012) Monocyte galactose/ $\mathrm{N}$-acetylgalactosamine-specific C-type lectin receptor stimulant immunotherapy of an experimental glioma. Part II: combination with external radiation improves survival. Cancer Manag Res 4 325-34.

98. He K, Qi Q, Chan CB, Xiao G, Liu X, et al. (2013) Blockade of glioma proliferation through allosteric inhibition of JAK2. Sci Signal 6: ra55

99. Panicker SP, Raychaudhuri B, Sharma P, Tipps R, Mazumdar T, et al (2010) p300- and Myc-mediated regulation of glioblastoma multiforme cell differentiation. Oncotarget 1: 289-303.

100. Chen J, Xu J, Zhao W, Hu G, Cheng H, et al. (2005) Characterization of human LNX, a novel ligand of Numb protein $X$ that is downregulated in human gliomas. Int J Biochem Cell Biol 37: 2273-2283.

101. Wu ZB, Cai L, Lin SJ, Lu JL, Yao Y, et al. (2013) The miR-92b functions as a potential oncogene by targeting on Smad3 in glioblastomas. Brain Res 1529 : $16-25$

102. Huber RM, Rajski M, Sivasankaran B, Moncayo G, Hemmings BA, et al. (2013) Deltex-1 activates mitotic signaling and proliferation and increases the clonogenic and invasive potential of U373 and LN18 glioblastoma cells and correlates with patient survival. PLoS One 8: e57793.

103. Lucio-Eterovic AK, Cortez MA, Valera ET, Motta FJ, Queiroz RG, et al (2008) Differential expression of 12 histone deacetylase (HDAC) genes in astrocytomas and normal brain tissue: class II and IV are hypoexpressed in glioblastomas. BMC Cancer 8: 243.

104. Yang W, Yang X, David G, Dorsey JF (2012) Dissecting the complex regulation of Mad4 in glioblastoma multiforme cells. Cancer Biol Ther 13: 1339-1348.

105. Saito N, Fu J, Zheng S, Yao J, Wang S, et al. (2013) A high Notch pathway activation predicts response to $y$ secretase inhibitors in proneural subtype of glioma tumor initiating cells. Stem Cells.
This article was originally published in a special issue, Signaling Pathways in Functional Cells handled by Editor(s). Dr. Li-Na Wei, University of Minnesota, USA 